\title{
PROBABILISTIC SITE IDENTIFICATION ANALYSIS USING NUPEC RECORDED FREE-FIELD MOTIONS
}

\author{
J. Xu, C. Costantino and C. Hofmayer \\ Brookhaven National Laboratory, Upton, New York 11973-5000, USA
}

\author{
A. Murphy \\ U.S. Nuclear Regulatory Commission, Washington, D.C. 20555-0001, USA \\ Y. Kitada \\ Nuclear Power Engineering Corporation, Tokyo, Japan
}

\begin{abstract}
This paper describes a probabilistic site identification analysis performed by BNL, using the free-field earthquake motions recorded at the NUPEC test site. The BNL analysis was intended to provide adequate characterization of the soil properties for the test site to be used for the SSI analyses. The free-field data were provided by NUPEC. The methodology employed in the BNL probabilistic analysis of site identification includes the Monte Carlo process in conjunction with equivalent linear convolution analyses for generating a large number of site profiles for use in convolution studies from which mean estimates of response can be generated. The random variable selected to characterize the site profile is the shear wave velocity in each soil layer of the site profile. A lognormal distribution was assumed with the standard deviation determined from available site data and applicable regulatory requirements. The convolution analyses were performed using an appropriate soil degradation model and the outcrop input motions generated from the recorded in-rock motions. The BNL analysis produced results in terms of the mean, median and various fractiles of free-field soil properties at the test site, and the corresponding surface response spectra, which are presented in this paper.
\end{abstract}

\section{INTRODUCTION}

Although significant improvements have been made in both theoretical characterization and numerical analysis of earthquakes and their impacts on structures, correlations are still difficult to be established between theoretical analyses and the limited data available from the response to actual earthquakes. In early 1990, the Nuclear Power Engineering Corporation (NUPEC) of Japan initiated a field test program aimed at the verification of the seismic analysis methods used in the nuclear facility design practice (Fukuoka, 1995; Suzuki, 2001). To achieve this objective, NUPEC has conducted a series of field tests (Kitada, 1999) with construction of scaled nuclear power plant (NPP) structures and the installation of free-field down-hole seismometers aimed at investigating various aspects of soil-structure interaction (SSI) effects, including embedment and dynamic structure-soil-structure interaction (SSSI) effects. As part of a collaborative agreement between the US Nuclear Regulatory Commission (NRC) and NUPEC, Brookhaven National Laboratory (BNL) performed various seismic response analyses using the NUPEC field test results and the industry standard methods. This paper describes the BNL probabilistic site identification analysis using NUPEC recorded free-field motions.

The test site is located in Aomori Prefecture in northern Japan, a region which experiences frequent seismic activities. Large-scale NPP models with dynamic characteristics similar to typical NPP structures were constructed on soils representative of actual NPP sites. Figure 1 shows a plan layout, which includes the three structural model configurations and two free-field locations. A number of seismometers were installed along the down-hole arrays at the two free-field locations as indicated in Figure 1. The site geological features consist of primarily weathered-pyroclastic and pyroclastic rocks overlain by a $5-8 \mathrm{~m}$ layer of overburden comprised of weathered sandstones and diluvial loams. The site water table is located between $7-10 \mathrm{~m}$ from the ground surface. Seismometer recordings for a number of earthquakes in the down-hole arrays at the two free-field locations were provided by NUPEC to BNL. The maximum free-field responses measured from these earthquakes were between 
8.9 gal to 174 gal, representing a reasonable range of energy transmissions through soils that can be used to calibrate both low strain free-field soil profiles and the strain dependent modulus degradations. The objective of the BNL free-field analysis is to identify the test site soil properties in terms of shear wave velocity using the seismometer recordings from these earthquakes.

The methodology employed in the BNL probabilistic analysis for site identification includes the Monte Carlo process in conjunction with equivalent linear convolution analyses. The random variable selected to characterize the site profile is the shear wave velocities in each : soil layer of the site profile. A lognormal distribution is assumed with the standard deviation determined from available site data and applicable regulatory. requirements. The median values are determined through the Fourier ratio method with least square fitting ( $\mathrm{Xu}$; 2001). The convolution analyses were performed using the CARES program (Xu, 1993) with the appropriate : soil degradation model and the outcrop input motions generated from : the : recorded in-column rock motions. The BNL analysis produced results in terms of the mean, : . median and various fractiles of free-field soil $\therefore$ properties at the test site, and the corresponding .: surface response spectra. In this paper, the method and procedure... employed to perform the probabilistic site analysis are : described first, $, \ldots, i$, , followed by the results of the BNL analysis with thirty randomly sampled profiles generated from one earthquake motion recorded at the NUPEC: site.

\section{ANALYSIS APPROACH}

Conventional probabilistic analysis consists of steps, including: 1) identification of random variables and their associated distributions; $; 2) \ldots$ modeling of probabilistic : process for developing various statistical data which characterize the random variables under consideration; 3) assessment of uncertainties associated with the : quantification of the random variables. In the site identification analysis, soil properties such as unit weight, damping; shear wave velocity $\left(\mathrm{V}_{\mathrm{s}}\right)$ and the thickness of soil layers all have inherent randomness in their measurements, and therefore could all be selected as random variables.: The probabilistic process established based on all soil properties and the associated uncertainties could be rather complicated and requires significant effort to simulate. However, since the SSI effect is : primarily influenced by the shear. wave velocity of soils, BNL selected $V_{s}$ to be the random variable in order to simply the analysis: The measurement $: \cdots$ of the other properties could be improved through the application of better techniques and instruments. The soil damping is characterized through the GEI modulus degradation model (GEI, 1983), which has been established to be suitable: for the NUPEC test site.

The BNL analysis assumes a lognormal distribution : for ' $V_{\mathrm{s}}$. The probability density function (PDF): of $V_{s}$, therefore, assumes the following form:

$$
f_{\mathrm{v}_{\mathrm{s}}}\left(v_{\mathrm{s}}\right) \doteq \frac{1}{\sqrt{2 \pi} \beta v_{s}} \exp \left[-\frac{1}{2}\left(\frac{\ln v_{s}-\ln v_{\text {sured }}}{\beta}\right)\right]
$$

where $\mathrm{v}_{\mathrm{smed}}$ represents the median estimate of $\mathrm{V}_{\mathrm{s}}$ and $\beta$ is the lognormal standard deviation of $: V_{s}$ characterizing the uncertainty for the $v_{\text {smed }}$. estimate. The low-strain $\mathbf{v}_{\text {smed }}$ is determined using the Fourier ratio method with a non-linear least square fitting technique: for correlating the analytical site amplification with the amplification computed using the recorded seismometer data. The BNL estimate $(\mathrm{Xu}, 2001)$ of the low: strain $\mathrm{v}_{\text {smed }}$ is shown in:Figure 2. The lognormal standard deviation is determined using the guidelines of the current version (NUREG-0800) of the USNRC Standard : Review Plan : (SRP). The SRP recommends that the $1: 5 \mathrm{G}$ and $\mathrm{G} / 1.5$ variations be used in SSI analyses to account for the free field soil uncertainties. Where, $G$ represents the best estimate of the shear modules of soils. The range of uncertainties determined by $1.5 \mathrm{G}$ and $\mathrm{G} / 1.5$ is about the 15 th to 85 th percentiles, corresponding to the mean $+\beta$ and mean- $\beta$ estimates. With the aid of the relation: $V_{s}=(G / \rho)^{1 / 2}$, and the following equation:

$$
\beta=\ln \frac{v_{.85}}{v_{m e d}},
$$

the lognormal standard deviation $\beta$ for.$V_{s}$ is: readily calculated to be 0.2 :

In order to apply the Monte Carlo method; random sampling of the low strain Vs of the free field: profile needs be performed. This is done by using. the uniform distribution for sampling and the 
cumulative distribution function (CDF) of $\mathrm{V}_{\mathrm{s}}$ with the lognormal model as given in Eq. (1). The set of free field sample profiles thus generated are each subsequently used in the convolution analyses, which in turn are iterated with a modulus degradation model and a rock outcrop input earthquake motion until the strain compatible soil properties are identified. The convolution analyses are performed using the CARES program (Xu, 1993) assuming a one-dimensional shear wave propagation. The results of this simulation process are a sample set of strain compatible free field profiles, including $V_{s}$, the corresponding final strains, and soil damping. The corresponding surface motions can also be calculated in terms of response spectrum, which may be used as input to SSI analyses.

\section{SAMPLE ANALYSIS}

BNL has performed the site identification analyses for the NUPEC site using the probabilistic approach as described in the previous section. Seven earthquakes recorded in the down-hole array of the free field were provided by NUPEC. Due to limited space, only the results for Earthquake No.139 are presented in this paper to demonstrate the application of the probabilistic approach to site identification. The results for other earthquakes will be documented in a NUREG report together with the SSI analyses when completed.

As described in the previous section, the median low strain free field profile was generated using the Fourier ratio method. This is shown in Figure 2 as the new free field point. The lognormal standard deviation of 0.2 was assumed based on the recommendation of the USNRC SRP. A set of randomly sampled profiles were then selected; BNL generated a total of thirty sample profiles. Figure 3 shows the low strain $V_{s}$ distributions for the median, mean, plus and minus $1 \beta$. It is shown in this figure that the mean profile, though slightly stiffer, is fairly close to the median, suggesting that the 30 samples are appropriate for this simulation.

Convolution analyses were then performed for each of the 30 low strain profiles and a rock outcrop. The rock outcrop was calculated by first convolving the in-column recorded rock motion to generate a surface motion. The surface motion was then deconvolved to generate a bedrock outcrop motion. Figure 4 shows the final strain compatible $\mathrm{V}_{\mathrm{s}}$ profiles in terms of the mean, and associated maximum and minimum $V_{s}$ for each layer. Since Earthquake No.139 is a small earthquake, with the maximum acceleration of about 9 gal induced at ground surface, it is expected that $V_{s}$ should stay in the low strain regime through the entire motion. This is evident in Figure 4, which shows that the iterated mean is coincident with the low strain mean. Figures 5 and 6 show the soil strains and the soil damping in terms of the mean, maximum and minimum. Figure 7 provides the surface response spectra plotted in terms of the mean, mean $+1 \beta$ and mean- $1 \beta$ together with the outcrop input. The surface mean response spectrum can then be used as input to an SSI analysis to compute the mean SSI response. One could also use a series of percentile spectra as inputs and propagate through the SSI analyses to generate a series of percentile SSI responses. A statistical analysis would then be performed to generate the mean response.

\section{CONCLUSIONS}

In this paper, a probabilistic method and its application procedure for site identification was presented in the context of a collaborative effort between BNL/NRC and NUPEC of Japan to perform a broad assessment of the industry practice for the SSI estimates of NPP structures. BNL has successfully applied the probabilistic approach and the current SRP recommendations to establish the mean free field profile and the mean surface response spectrum. These free field properties will then be used in the SSI analyses as part of the collaboration. This paper, through a complete sample analysis for one earthquake, has demonstrated the feasibility of the probabilistic approach in determining the free field soil properties.

\section{REFERENCES}

Fletcher R., 1971 "A modified Marquadt Subroutine for Nonlinear Least Square Records," Harwell Report, AERE-R, 6799.

Fukuoka, A, 1995, "Dynamic Soil-Structure Interaction of Embedded Structure," Trans. $13^{\text {th }}$ SMiRT, Vol. III, Port Alegre.

Geotechnical Engineers Inc (GEI), 1983, "Evaluation of Dynamic Soil Properties for F-Area Sand Filter Structures," Report Prepared for E.I. Dupont de Nemours \&Co.

Kitada, Y., et al., 1999, "Model Test on Dynamic Structure-Structure Interaction of Nuclear Power Plant Buildings," Nuclear Engineering and Design (192), 205-216. 
Kurita, T., 1997; "Dynamic Characteristics of Soil Deposits Identified from Seismic Records," . Trans. $14^{\text {th }}$ SMiRT, Lyon, France.

Suzuki, A. et al., 2001, "Evaluation of Seismic Input Motions and Responses of Buildings in Nuclear Power : Plants, $\cdots$ Proceedings of the OECD/NEA $\cdots$ Workshop $:$ on $\cdots$ the Engineering Characterization: of Seismic Input, Brookhaven National Laboratory,... Upton, New .... York, NEA/CSNI/R(2000)2/Volume. 2.

$\mathrm{Xu}$, J., et. al., 2001 ,, "Identification of Free-Field Soil Properties. Using NUPEC Recorded Ground Motions,", Trans. $16^{\text {th }}$. SMiRT, Washington, D.C., U.S:A
Xu, J., et al.,; 1993, "Integrated Software System for Seismic : Evaluations of Nuclear Power Plant Structures," The :- International :: Journal of Computers \& Structures; Vol.46, No.4, pp.717723.

\section{NOTICE}

This work was performed under the auspices of the U.S." Nuclear Regulatory Commission, Washington,, D.C. The findings and opinions expressed in this paper are those of the authors, and do not necessarily reflect the views of the.U.S. Nuclear - Regulatory Commission; Brookhaven National Laboratory or the . Nuclear Power Engineering Corporation.

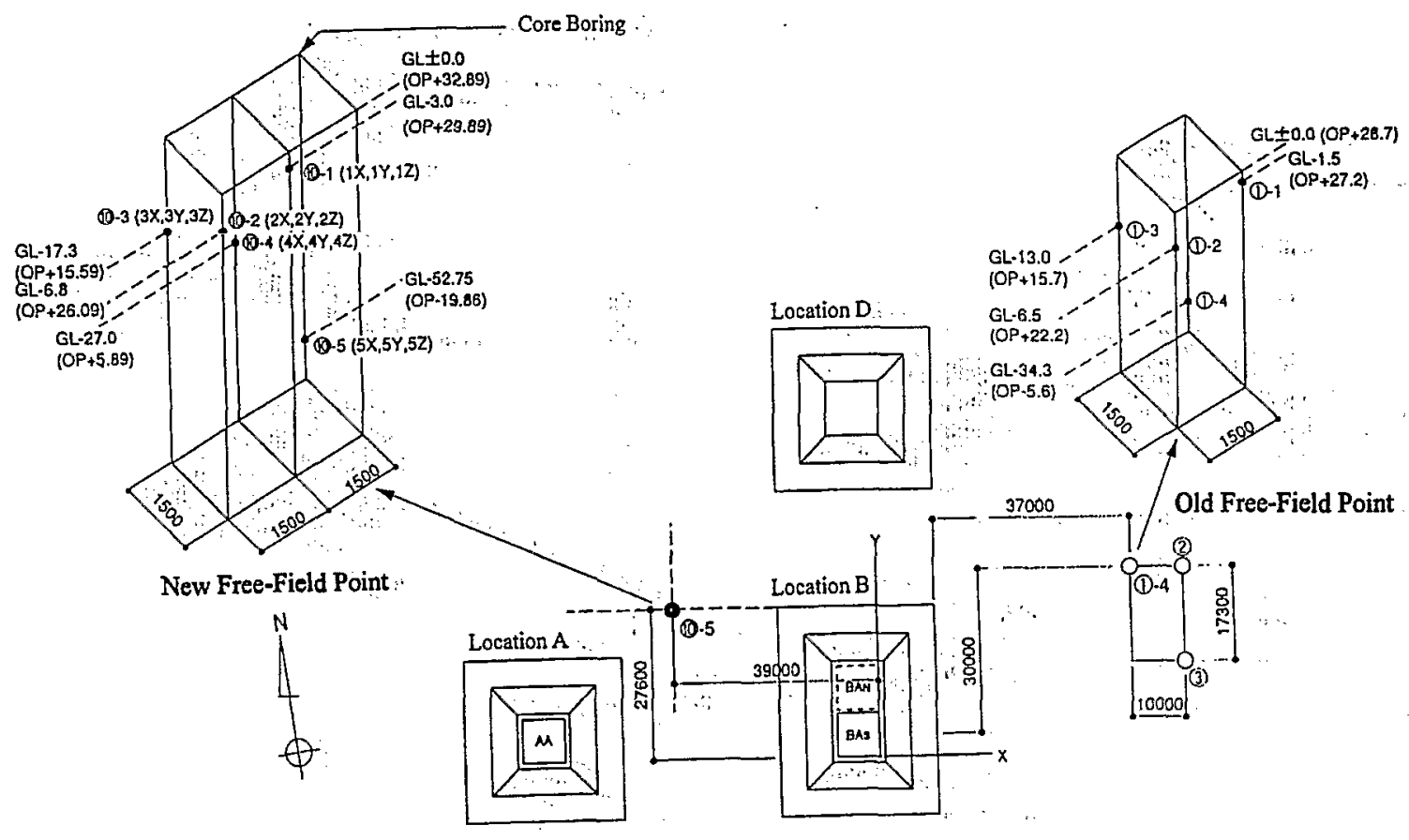

Figure 1. Layout of Seismometers in Free-Field Down-Hole Arrays. 


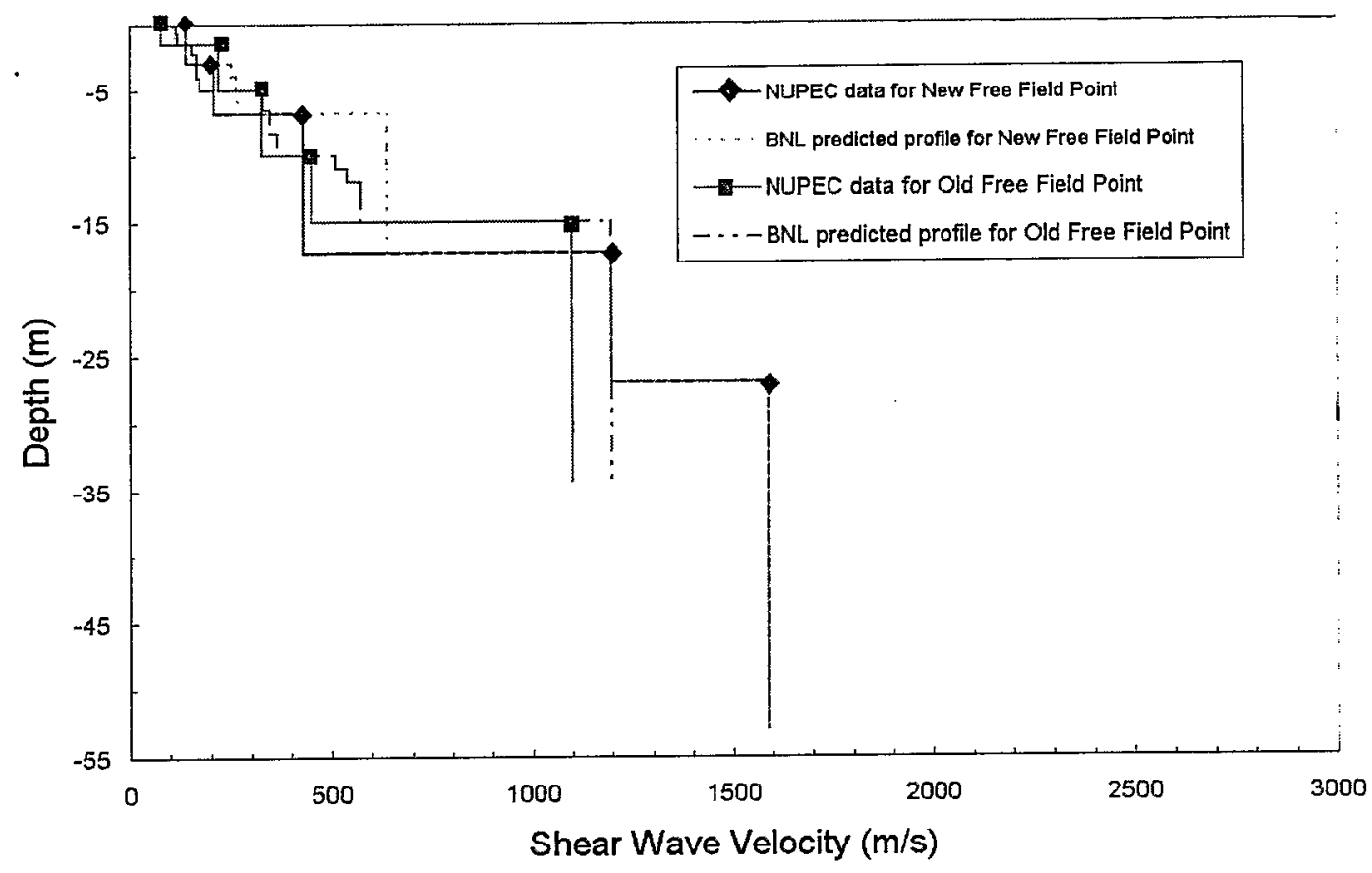

Figure 2. BNL predicted low strain median profiles for New/Old Free Field Points.

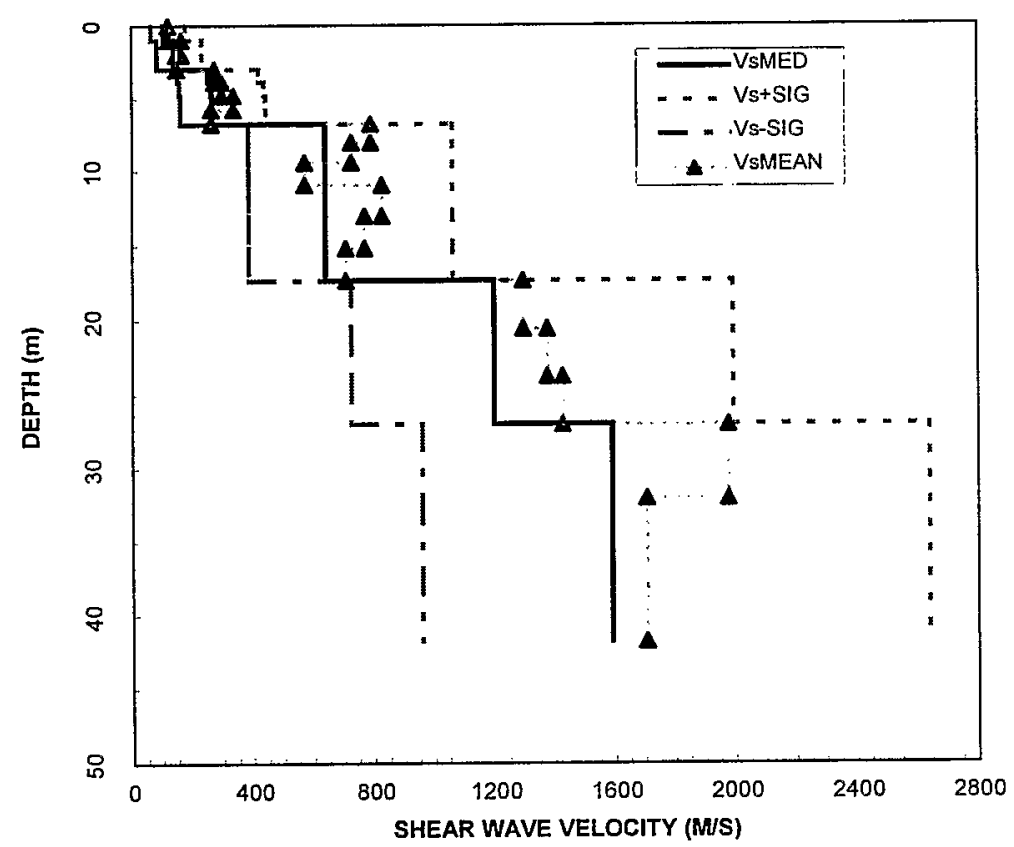

Figure 3. Low strain soil profile distribution. 


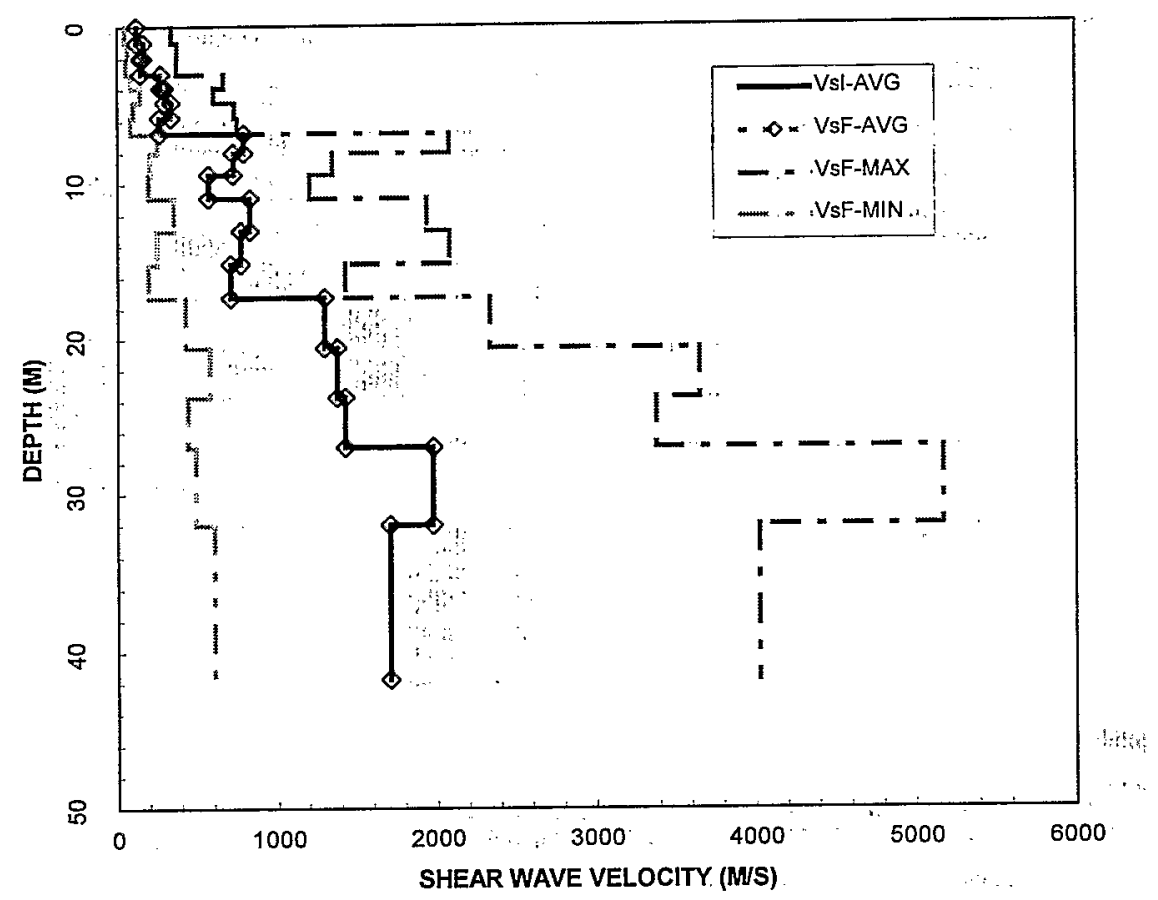

Figure 4. Range of Iterated soil profiles for Earthquake 139x. "

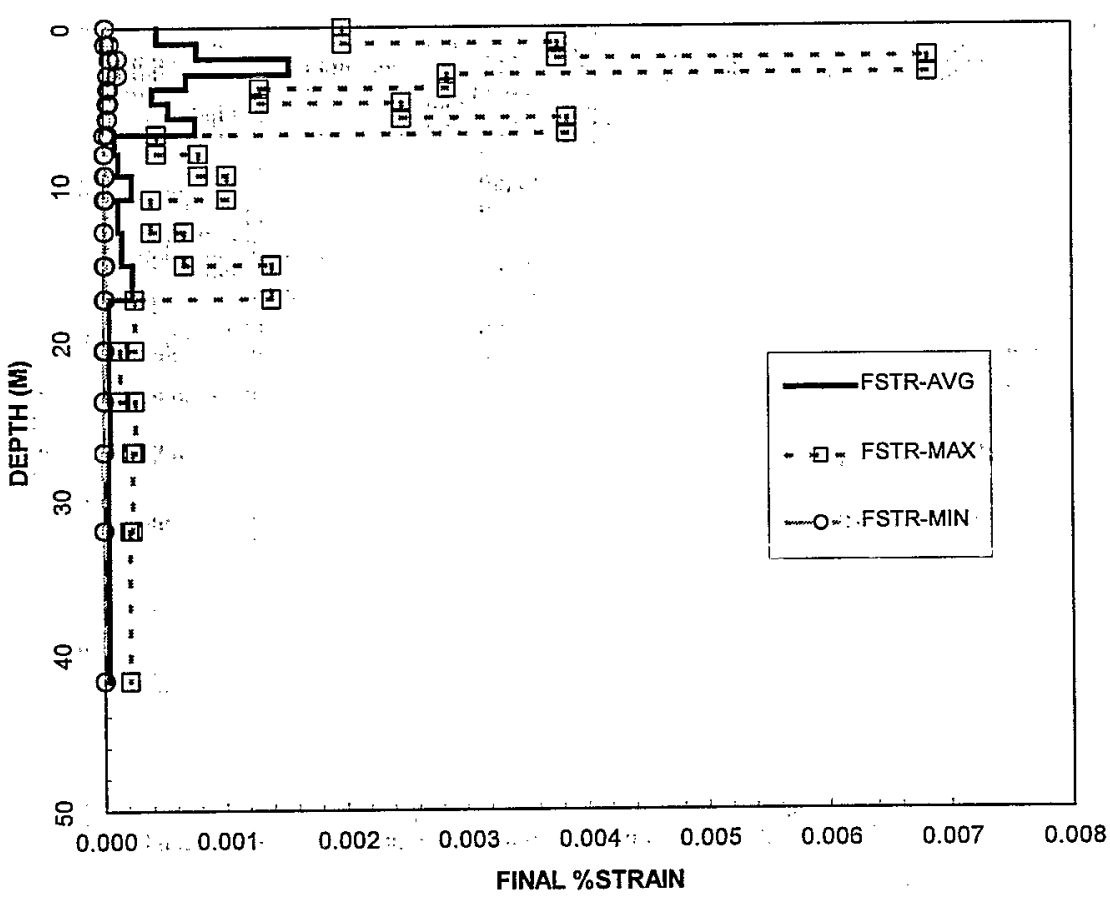

Figure 5. Range of Iterated shear strains: (\%) for Earthquake 139x. 


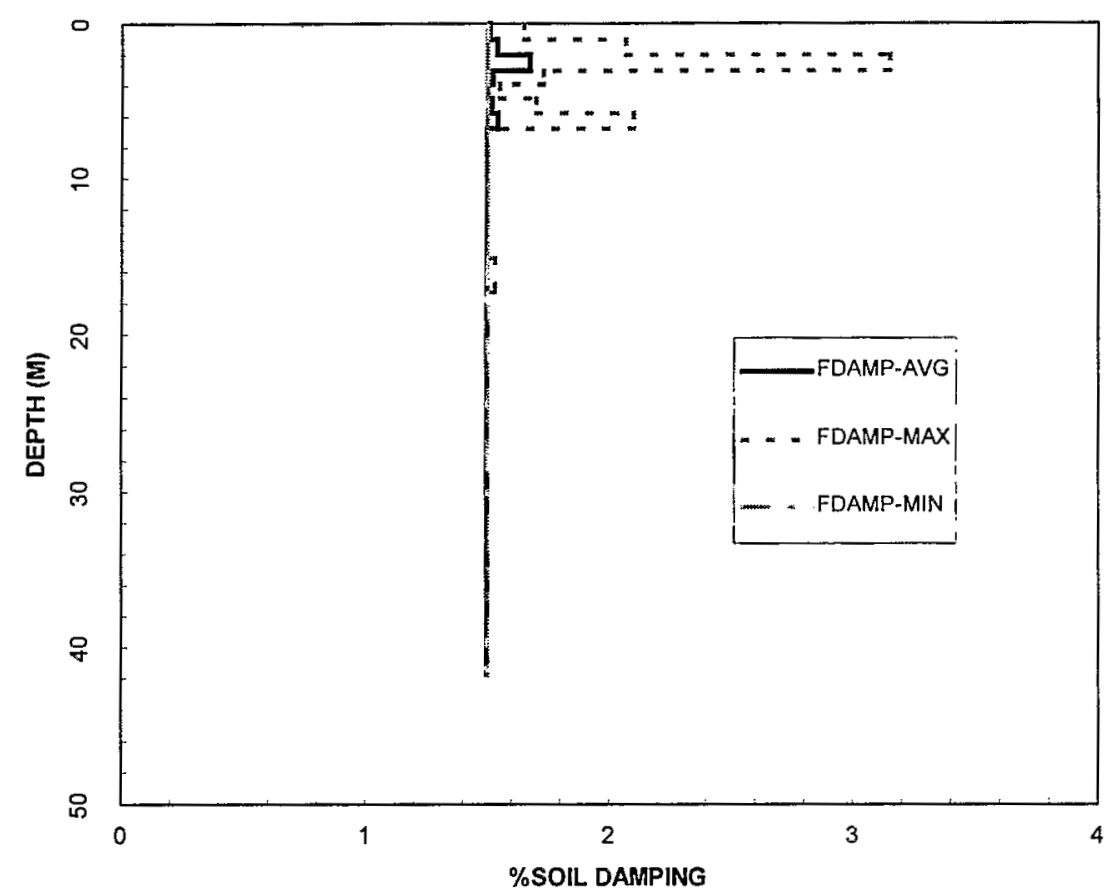

Figure 6. Range of Iterated soil damping (\%) for Earthquake 139x.

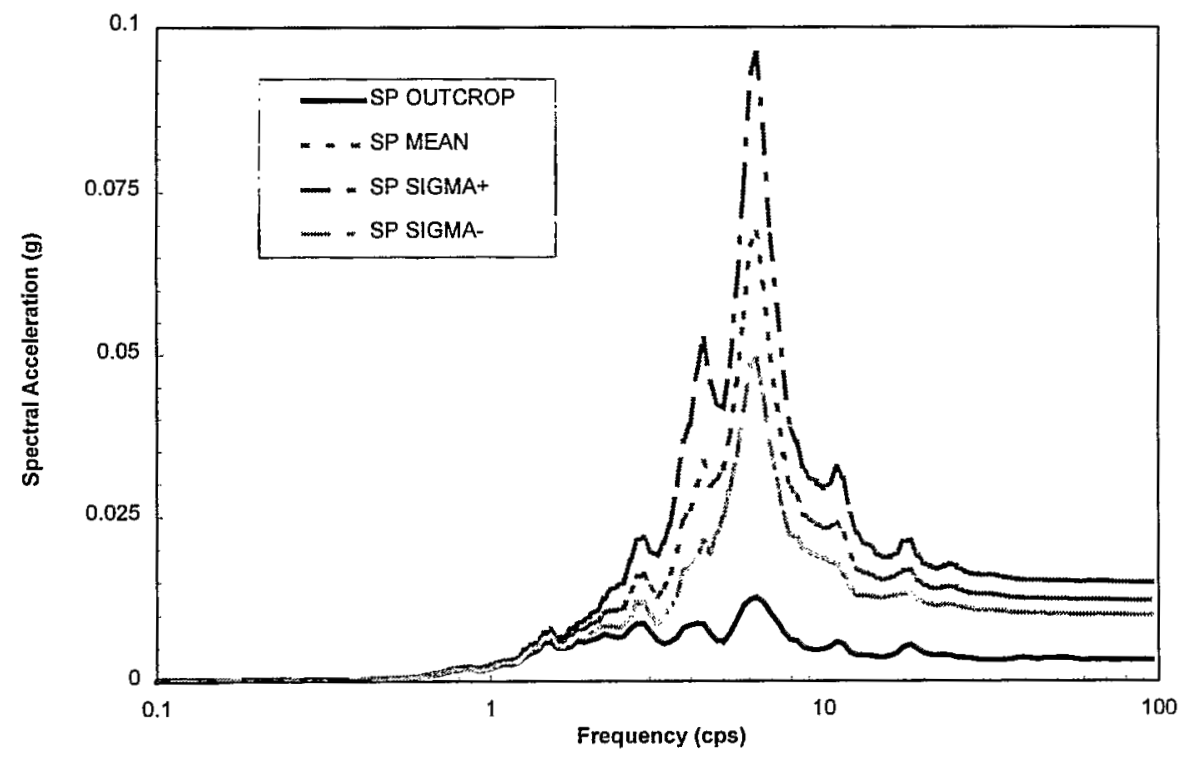

Figure 7. Generated surface spectra for Earthquake 139x. 